\title{
Network Tomography via Compressed Sensing
}

\author{
Mohammad H. Firooz, Student Member, IEEE, Sumit Roy Fellow, IEEE, \\ Electrical Engineering Department \\ University of Washington \\ Seattle, WA 98105. \\ \{firooz,sroy\}@u.washington.edu
}

\begin{abstract}
In network tomography, we seek to infer link parameters inside a network (such as link delays) by sending end-to-end probes between (external) boundary nodes. The main challenge here is to estimate link-level attributes from end-to-end measurements. In this paper, based on the idea of combinatorial compressed sensing, we specify conditions on network routing matrix under which it is possible to estimate link delays from measurements of end-to-end delay. Moreover, we provide an upper-bound on the estimation error.
\end{abstract}

\section{INTRODUCTION}

The monitoring of link properties within a network (such as delays and loss rates) has been stimulated by the demand from network engineers and Internet Service Providers (ISP) for network management tasks. For instance, fault and congestion detection or traffic monitoring would help to keep track of network utilization and performance. The need for accurate and fast network monitoring methods has increased further in recent years due to the complexity of new services (such as video-conferencing, Internet telephony, and on-line games) that require high-level quality-of-service (QoS) guarantees. In 1996, the term network tomography was coined by Vardi [1] to encompass this class of methods that seek to infer internal link parameters and identify link congestion status.

Current network tomography methods can be broadly categorized as follows [2]:

- Node-oriented: These methods are based on cooperation among network nodes on an end-to-end route using control packets. For example, active probing tools such as ping or traceroute, measure and report attributes of the round-trip path (from sender to receiver and back) based on separate probe packets[3]. The challenges of such node-oriented methods arise from the fact that many service providers do not own the entire network and hence do not have access to the internal nodes[4].

- Path-oriented: In networks with a defined boundary, it is assumed that access is available to all nodes at the edge (and not to any in the interior). A boundary node sends probes to all (or a subset) of other boundary nodes to measure packet attributes on the path between network end-to-end points. Clearly, these edge-based methods do not require exchanging special control messages between interior nodes. The primary challenge of such end-to-end probe data [5],[6] to estimate link level attribute is that of identifiability, as will be discussed later.
As the Internet evolves towards decentralized, uncooperative, heterogeneous administration and edge-based control, node-oriented tools will be limited in their capability. Accordingly, in this work, we only focus on path-oriented methods which have recently attained more attention due to their ability to deal with uncooperative and heterogeneous (sub)networks.

In path-oriented network tomography, probes are sent between two boundary nodes on pre-determined routes; these are typically the shortest paths between the nodes. For some parameters such as delay, which is the main concern of this manuscript, a linear model can adequately capture the relation between path delays (from end-to-end measurements) and individual link delays, and can be written as [7], [8]

$$
\mathbf{y}=\mathbf{R x}
$$

where $\mathbf{x}$ is the $n \times 1$ vector of individual link delays. The $r \times n$ binary matrix $\mathbf{R}$ denotes the routing matrix for the network graph, and $\mathbf{y} \in \mathbb{R}^{r}$ is the measured $r$-vector of endto-end path delays. Solution approaches based on Eq. (1) can be categorized as follows:

1) Deterministic models: Here the link attributes, such as link delay, are considered as unknown but constant; the goal of network tomography is to estimate the value of those constants. Since the link delay is typically time varying in any network, this approach is suitable for periods of local 'stationarity' where such an assumption is valid.

2) Stochastic model: Here, it is supposed that the link vector $\mathbf{x}$ is specified by a suitable probability distribution. The goal of network tomography is to identify the unknown parameters of the probability model. For example, many works assume the link attributes follow a Gaussian distribution or an exponential distribution [9], [7], [8]. Further, the observations are assumed to occur in the presence of an independent additive noise or interference term $\epsilon$ [10]; thus the observation equation is modified to $\mathbf{y}=\mathbf{A x}+\epsilon$.

There exist challenges with both modeling approaches. Stochastic approaches in literature are Bayesian in nature, requiring a prior distribution. If incorrectly chosen, this can lead to biases in the resulting estimations. Furthermore, stochastic models are usually more computationally intensive than deterministic ones [11]. On the other hand, deterministic models, the one we conform to, suffer from generic identifiability problems; this will be discussed subsequently in more detail. 
In Eq. (1), typically, the number of observations $r \ll n$, because the number of accessible boundary nodes is much smaller than the number of links inside the network. Thus the number of variables in Eq. (1) to be estimated is much larger than number of equations in the linear model $\operatorname{rank}(\mathbf{R})<$ $n)$ [10], leading to generic non-uniqueness for any solution to Eq. (1); in other words, it is impossible to uniquely specify link delays [9].

A network administrator is typically interested in identifying only the (few) links with large delays (or high packet lost rate) at any given time; this information allows a pathway to solve the underdetermined system in Eq. (1) provided that the sparsity can be exploited. In other words, that we are interested in identifying solution vectors $\mathbf{x}$ with only a few large entries (say, up to $k$ ). We refer to such vectors as $k$-sparse vector. We will show that by using the concept of expander graphs and compressed sensing, $k$-sparse delay vectors may be successfully estimated, provided some conditions on the routing matrix of a network are met. The estimates obtained satisfy a desirable property, i.e. the difference between the true delay and the estimate (solution from Eq. (1)) goes to zero. We call such networks $k$-identifiable. In addition, we show that if network is $k$-identifiable, Eq. (1) can be solved using a LP optimizer.

Our specific contributions in this work are summarized next:

- We establish a connection between network tomography and binary compressed sensing using expander graphs which has received significant interest during the past few years.

- We provide conditions on the routing matrix of networks for which the network is $k=1$-identifiable.

- We provide an upper-bound on estimation error on link delay when network in 1-identifiable.

As is customary, a network consisting of bidirectional links connecting transmitters, switches, and receivers can be modeled as an undirected graph $N(V, E)$ where $V$ is the set of all vertices (nodes) and $E$ the set of all edges (links). Let $B \subset V$ be a subset of accessible boundary nodes that can act as probe sources and sinks; a set of measurements $\mathbf{y}$ are obtained by end-to-end probing, given by Eq. (1).

The paper is organized as follows: Section II relates routing matrix of a network to bipartite graphs. Section III relates links delay estimation to binary compressed sensing and gives condition on network routing matrix under which a given network is 1-identifiable. While this is the simplest possible class of identifiability problems (compared to the general $k>1$ case), it is sufficiently illuminating as our investigations will show.The paper concludes with reflections on future work in Section IV.

Notations: We use bold capitals (e.g. R) to represent matrices and bold lowercase symbols (e.g. $\mathrm{x}$ ) for vectors. The $i$-th entry of a vector $\mathbf{x}$ is denoted by $x_{i}$ and Superscript ${ }^{t}$ shows matrix transpose. A set is denoted by a calligraphic capitalized symbol, e.g. $\mathcal{R}$.

For any set $S \subset\{1,2,3, \ldots n\}$, we use $S^{c}$ to denote the complement of $S$. Also, for any vector $\mathbf{x} \in \mathbb{R}^{n}$, vector $\mathbf{x}_{S}$ is

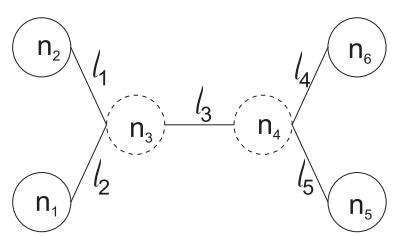

Fig. 1. A network with 4 boundary nodes, 2 intermediate nodes and 5 links

a vector with entries defined as follows:

$$
\left(x_{S}\right)_{i}= \begin{cases}x_{i} & \text { if } i \in S \\ 0 & \text { o.w. }\end{cases}
$$

\section{ROUTING MATRIX AND BIPARTITE GRAPH}

In this section we show how the routing matrix of a network can be interpreted as a bi-adjacency matrix of a suitably defined bipartite graph. This helps make a connection between the notion of network identifiability and expander graphs, a subset of bipartite graphs. In Figure 1, a toy network with 4 boundary nodes and 2 intermediate nodes is depicted. Throughout this work, boundary nodes are depicted as solid circles while intermediate nodes are presented using dashed circle.

A bipartite graph is one whose vertices can be divided into two disjoint sets $X$ and $Y$ such that every edge connects a vertex in $X$ to one in $Y$. A bipartite graph is usually presented as a triple $G(X, Y, H)$ where $H \subset X \times Y$ is a set of edges between two parts. Sets $X$ and $Y$ are called left side and right side of the graph, respectively. A bipartite graph $G(X, Y, H)$ can also be represented with a matrix $\mathbf{A}=\left[a_{i j}\right]$, known as bi-adjacency matrix, where $a_{i j}=1$ if node $i$ in $\mathrm{X}$ is connected to node $j$ in $Y$, or equivalently if $(i, j) \in H$ and it is zero otherwise.

Suppose a network $N(V, E)$ is given. Let $n$ be the number of links in this network $(n=|E|), \mathcal{R}$ the given collection of paths between boundary nodes, where $r$ is the cardinality of $\mathcal{R}$ (i.e., total number of paths between boundary nodes). Further, let $\mathbf{R}_{r \times n}$ be the routing matrix corresponding to the set $\mathcal{R}$. These are equivalent in the sense of containing the same information about existing paths between boundary nodes inside the network.

For the network in Figure 1, suppose the following routing matrix is given:

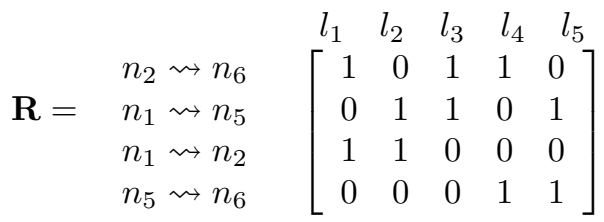

which corresponds to collection of paths $\mathcal{R}$ as follows:

$$
\mathcal{R}=\left\{l_{1} l_{3} l_{5}, l_{2} l_{3} l_{4}, l_{1} l_{2}, l_{4} l_{5}\right\}
$$

Note that the above routing matrix or the equivalent set of paths is not a complete routing matrix of network in Figure 1. For instant it doesn't include the path from $n_{1}$ to $n_{6}$ which is $l_{2} l_{3} l_{4}$. However, a fundamental underlying assumption in 


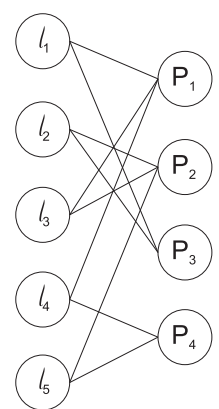

Fig. 2. bipartite graph corresponding to given routing matrix in Eq. (3)

network tomography is that the routing matrix is fixed and may not be changed; the goal is to use this given routing matrix to estimate links parameters (delay is our case) inside the network. The design problem, i.e. where the selection of paths (equivalently choice of routing matrix) is a free variable such that the network is 1-identifiable, is left for future investigation.

$\mathbf{R}_{r \times n}$ can be thought of a bi-adjacency matrix of a bipartite graph $G(X, Y, H)$ where $X=E$, set of links in network $N(V, E)$, and $Y=\mathcal{R}$, the set of given paths in the network. A node in $X$ is connected to a node in $Y$ if the path in $Y$ goes through the corresponding link in $X$. Figure 2 presents the corresponding bipartite graph of network in Figure 1 with routing matrix $\mathbf{R}$ in Eq. (3).

\section{EXPANDER GRAPH AND Network IDENTIFIABILITY}

In this section we establish a connection between identifiability in a network $N(V, E)$ to the recently developed concept of compressed sensing using expander graphs.

\section{A. Expander Graphs}

In recent years, a new approach for obtaining a succinct approximate representation of $n$-dimensional vectors (or signals) has gained significant attention - compressive sensing [12], [13], [14]. For any signal $\mathbf{x}$, the representation available is equal to $\mathbf{A x}$, where the measurement matrix $\mathbf{A}$ has dimension $m \times n$ matrix $(m<<n)$. The traditional reconstruction problem in compressive sensing is to obtain an estimate of $\mathbf{x}$ from this lower-dimensional or compressed version, given some side information about $\mathbf{x}$, notably it's sparsity, i.e. the number of significant (non-zero) entries.

The main challenge is to embed $\mathbf{A}$ with desirable properties (assuming it to be a design variable), such as maximum possible compression or it's ability to support fast approximate recovery efficiently, subject to error bounds)[15], [16], [17], [18]. However the problem we face here is the dual problem; since the measurement matrix (equivalently, the routing matrix) is already pre-determined, the question to be answered is whether the (given) routing matrix supports compressive sensing. To the best of the authors knowledge, there is no study in the literature regarding this.

The measurement matrix in tomography is a binary matrix that can be considered as a bi-adjacency matrix of a bipartite graph. Berinde and Indyk in [19] show that bi-adjacency matrix of special bipartite graphs, called expander graphs, can be used as measurement matrix.

Definition 1. A $(\phi, d, \epsilon)$-expander is a bipartite simple graph $G(X, Y, H)$ with left degree d (i.e. $\operatorname{deg}(v)=d \forall v \in X$ ) if for any $\Phi \subset X$ with $|\Phi| \leq \phi$ the following condition holds:

$$
|N(\Phi)| \geq(1-\epsilon) d|\Phi|
$$

where $N(\Phi)$ is set of neighbors of $\Phi$. Parameters $\phi$ and $\epsilon$ are referred to as "expansion factor" and "error parameter", respectively.

Roughly speaking, in an expander graph, the degree of any set of nodes in the left hand side $(X)$ is 'expansive', i.e. they connect to a sufficiently large number of nodes in the right hand side $(Y)$. Expander graphs are well-studied in the literature with a number of works describing how to construct such a $(\phi, d, \epsilon)-$ expander graph. Interested readers may refer to [20], [21], [22].

Berinde and Indyk in [23], [19] show that bi-adjacency matrix of a $(2 \phi, d, \epsilon)$ - expander graph can be used as measuring matrix for a $\phi$-sparse signal. Therefore, to show a given network $N(V, E)$ with routing matrix $\mathbf{R}$ is 1-identifiable it is enough to show that the corresponding bipartite graph with bi-adjacency matrix $\mathbf{R}$ is a 2-expander graph. The error $\epsilon$ of an expander graph is a design parameter which is related to recovery error from the compressive measurements. Berinde and Indyk derive their results for $\epsilon<\frac{1}{16}$. However, in order to potentially identify as large a class of (binary) measurement matrices, we choose $\epsilon$ to be as large as possible, at the expense of increasing the re-construction error. In other words, higher $\epsilon$ expands the space of expander graphs which in turn map to routing matrices that are 1-identifiable. We next quote a theorem (the proof is omitted due to space constraints) characterizing our key result and show via an example that the bipartite graph in Figure 2 is an expander graph for $\epsilon=1 / 4$.

Theorem 1. Let $G(X, Y, E)$ be a $(2, d, \epsilon)$-expander graph with bi-adjacency matrix $\mathbf{A}$. Further assume $\mathbf{w}$ lies in null space $\mathbf{A}(\mathbf{A w}=\mathbf{0})$ and let $S$ be any set of $k=1$ coordinates of $\mathbf{w}$. Then

$$
\left\|\mathbf{w}_{S}\right\|_{1} \leq 2 \epsilon\left\|\mathbf{w}_{S^{c}}\right\|_{1}
$$

proof: See [24].

The following theorem places an upper bound on error of recovering $\mathbf{x}$ from its linear projection $\mathbf{A x}$ when $\mathbf{A}$ is a biadjacency matrix of an $(2, d, \epsilon)$-expander graph.

Theorem 2. Consider any two vectors $\mathbf{x}, \mathbf{x}^{\prime}$, such that they have the same projection under measurement matrix $\mathbf{A}$; i.e. $\mathbf{A x}=\mathbf{A x}^{\prime}$. Further, suppose $\left\|\mathbf{x}^{\prime}\right\|_{1} \leq\|\mathbf{x}\|_{1}$. Let $S$ be the set of $k=1$ largest (in magnitude) coefficients of $\mathrm{x}$. Then

$$
\left\|\mathbf{x}^{\prime}-\mathbf{x}\right\|_{1} \leq f(\epsilon)\left\|\mathbf{x}_{S^{c}}\right\|_{1}
$$

where $f(\epsilon)=\frac{1+2 \epsilon}{2(1-2 \epsilon)}$ 
Illustrative Example: We show that the routing matrix of network depicted in Figure 1 can be used as a measurement matrix, as it is the bi-adjacency matrix of the bipartite graph in Figure 2 and this bipartite graph is a $(2,2,1 / 4)$-expander graph. For instance, let $\Phi=\left\{l_{1}, l_{2}\right\}$. In that case, $N\left(\left\{l_{1}, l_{2}\right\}\right)$ which is the set of nodes connected to $\left\{l_{1}, l_{2}\right\}$ is:

$$
N\left(\left\{l_{1}, l_{2}\right\}\right)=\left\{P_{1}, P_{2}, P_{3}\right\}
$$

implying that $\left|N\left(\left\{l_{1}, l_{2}\right\}\right)\right|=3$ and inequality (5) holds as equality.

The null space of matrix in Eq. (3), having dimension one, is given as below:

$$
\mathbf{w}=\left[\begin{array}{lllll}
1 & -1 & 0 & -1 & 1
\end{array}\right]^{t}
$$

Since $\epsilon=1 / 4$, by Theorem 1 , the following inequality holds for bi-adjacency matrix of bipartite graph in Figure 2:

$$
\left\|\mathbf{w}_{S}\right\|_{1} \leq 0.5\left\|\mathbf{w}_{S^{c}}\right\|_{1}
$$

For example, let $S=\{1\}$. Then $\mathbf{w}_{S}$ and $\mathbf{w}_{S^{c}}$ can be written as follows:

$$
\mathbf{w}_{S}=\left[\begin{array}{c}
1 \\
0 \\
0 \\
0 \\
0
\end{array}\right] \quad \mathbf{w}_{S^{c}}=\left[\begin{array}{c}
0 \\
-1 \\
0 \\
-1 \\
1
\end{array}\right]
$$

Thus, $\left\|\mathbf{w}_{S}\right\|_{1}=1$ and $\left\|\mathbf{w}_{S^{c}}\right\|_{1}=3$ and inequality (10) is satisfied.

Assume that the (unknown) true delay vector is

$$
\mathbf{x}=\left[\begin{array}{lllll}
0.1 & 0.1 & 1 & 0.1 & 0.1
\end{array}\right]^{t}
$$

Then the measured vector using the measurement matrix in (3) equals

$$
\mathbf{R x}=\left[\begin{array}{llll}
2.2 & 2.2 & 0.2 & 0.2
\end{array}\right]^{t}
$$

Since $\mathbf{A w}=\mathbf{0}$, the set of all possible solutions is

$$
\mathbf{x}^{\prime}=\mathbf{x}+\alpha \mathbf{w}=\left[\begin{array}{c}
.1 \\
.1 \\
1 \\
.1 \\
.1
\end{array}\right]+\alpha\left[\begin{array}{c}
1 \\
-1 \\
0 \\
-1 \\
1
\end{array}\right]
$$

For $\alpha=.05$, the corresponding estimate $\mathbf{x}^{\prime}$ is

$$
\mathbf{x}^{\prime}=\left[\begin{array}{lllll}
0.125 & 0.075 & 1 & 0.075 & 0.125
\end{array}\right]^{t}
$$

Since $\epsilon=1 / 4$, we have $f(\epsilon)=1.5$. Let $S=\{3\}$, then from Theorem 2 we have:

$$
\begin{aligned}
\left\|\mathbf{x}^{\prime}-\mathbf{x}\right\|_{1}=.1 & \leq f(\epsilon)\left\|\mathbf{x}_{S^{c}}\right\|_{1} \\
& =1.5 *(.1+.1+.1+.1) \\
& =.6
\end{aligned}
$$

\section{B. Delay Estimation}

The following theorem shows how to estimate delay in a given network $N(V, E)$ using the results of expander graphs. In addition, it proves that end-to-end measurement equation in (1) can be solved using a LP optimizer.

Theorem 3. Let $N(V, E)$ be a network with set of paths $\mathcal{R}$ and corresponding routing matrix $\mathbf{R}$. Suppose $G(E, \mathcal{R}, H)$ is a bipartite graph with bi-adjacency matrix $\mathbf{R}$. Assume $\mathbf{x}^{*}$ is the true delay vector of $N(V, E)$. Let $\mathbf{x}$ be a solution to the following LP optimization:

$$
\begin{aligned}
& \min \|\mathbf{x}\|_{1} \\
& \text { s.t. } \\
& \mathbf{R x}=\mathbf{R} \mathbf{x}^{*}
\end{aligned}
$$

Then

$$
\left\|\mathbf{x}-\mathbf{x}^{*}\right\|_{1} \leq f(\epsilon)\left\|\mathbf{x}_{S^{c}}\right\|_{1}
$$

if $G$ is a $(2, d, \epsilon)$-expander with $\epsilon \leq \frac{1}{4}$.

proof: See [24].

First note that if the true delay vector $\mathbf{x}$ is 1-sparse, it implies $\left\|\mathbf{x}_{S^{c}}\right\|_{1}=0$ which means $l_{1}$-norm minimization in Eq. (18) can recover signal completely with zero estimation error. However, if the true delay vector contains links with small but non-zero delays (the more likely scenario), then the estimation error is not zero and the above theorem places an upper bound on estimation error.

It should be mentioned that in general, the reverse of Theorem 3 is not true; i.e. there exist networks $N(V, E)$ which are 1-identifiable but their corresponding bipartite graph is not an expander graph. An example of such a graph is depicted in Figure 3-a. The bipartite graph of its routing matrix is presented in Figure 3-b. As can be seen, this bipartite graph is not regular (the degree of a node in left set is either one or two) in its left side which means it cannot be an expander graph (note: regularity is part of definition of an expander graph). However, Figure 4-(a) and (b), respectively, present subgraphs of $G$ with regular left degree one and two and each of these subgraphs is an expander.

The above observation suggests the extension in Theorem 3 for networks whose corresponding bipartite graph is not regular (and therefore not expander) but consists of expander sub-graphs.

Theorem 4. Let $N(V, E)$ be a network with routing matrix R. Let $G(X, Y, H)$ be a bipartite graph with bi-adjacency matrix R. Suppose $G_{i}\left(X_{i}, Y, H_{i}\right), i=1,2, \ldots M$ be $d_{i}$-regular bipartite subgraphs of $G$ such that:

- $X=\cup X_{i}$

- $H=\cup H_{i}$

- $d_{i} \neq d_{j}$ for $i \neq j$

Then, $N(V, E)$ is 1-identifiable, if each of $G_{i}$ is an $\left(2, d_{i}, \epsilon\right)$ expander graph for $\epsilon \leq \frac{1}{4}$. Further, link delays may be estimate as the solution to LP optimization in Eq. (18)

proof: see [24]. 


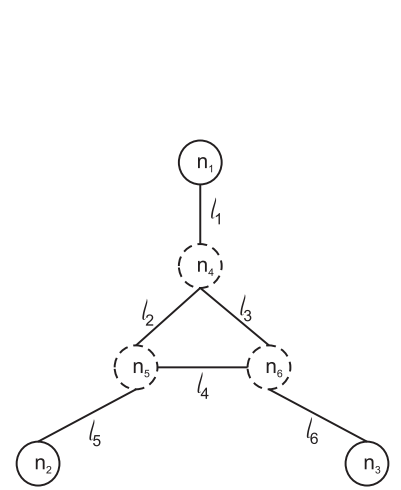

(a)

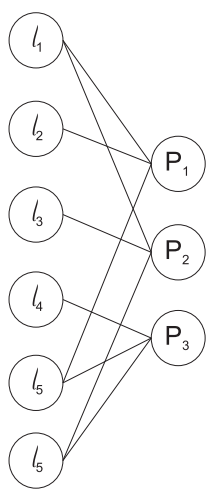

(b)
Fig. 3. An example of network which is 1-identifiable but its corresponding bipartite graph is not an expander graph (a) Network topology (b) Its corresponding bipartite graph

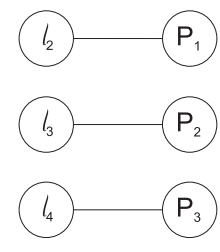

(a)

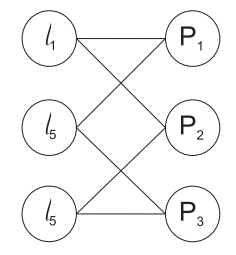

(b)
Fig. 4. Two subgraphs of bipartite graph in Figure 3-b which are regular in their left side

Basically, the above theorem says that a network $N(V, E)$ with routing matrix $\mathbf{R}$ is 1-identifiable if every two links $l_{i}$ and $l_{j}$ in the network either a) have different degrees in bipartite graph $G$ (meaning they belong to different expander graphs) or b) they satisfy the expansion property in Eq. (5).

\section{CONCLUSION}

This work presented a novel approach for estimating internal link delays in a network from end-to-end measurements obtained via probing between boundary nodes. Using the idea of binary compressed sensing, which has received significant attention in the past few years, we provide both a method for such identification and an upper bound on recovery error. Sending probes between nodes on the boundary of a network clearly comes with the cost of increasing traffic inside the network. How to design the routing matrix of a given network to minimize the number of injected probes needed while the network remains 1-identifiable remains an important future work.

\section{REFERENCES}

[1] Y. Vardi, "Network tomography: Estimating source-destination traffic intensities from link data," Journal of the American Statistical Association, vol. 91 , no. 433, 1996.

[2] E. Lawrence, G. Michailidis, V. Nair, and B. Xi, "Network tomography: a review and recent developments," Ann Arbor, vol. 1001, pp. 48 1091107.

[3] S. W. Richard, "TCP/IP illustrated," Addison-Welsey Publishing Company, 1994
[4] Y. Bejerano and R. Rastogi, "Robust monitoring of link delays and faults in IP networks," in Twenty-Second Annual Joint Conference of the IEEE Computer and Communications Societies (INFOCOM'03), vol. 1, 2003, pp. 134-144.

[5] R. Caceres, N. Duffield, J. Horowitz, and D. Towsley, "Multicastbased inference of network-internal loss characteristics," IEEE Trans. Information theory, vol. 45, no. 7, pp. 2462-2480, 1999.

[6] Y. Tsang, M. Coates, and R. Nowak, "Passive network tomography using em algorithms," in Proc. IEEE Int. Conf. Acoustics, Speech, and Signal Processing(ICASSP'01), vol. 3, 2001.

[7] A. Coates, A. Hero III, R. Nowak, and B. Yu, "Internet tomography," IEEE Signal processing magazine, vol. 19, no. 3, pp. 47-65, 2002.

[8] T. Bu, N. Duffield, F. Presti, and D. Towsley, "Network tomography on general topologies," ACM SIGMETRICS Performance Evaluation Review, vol. 30, no. 1, pp. 21-30, 2002.

[9] Y. Xia and D. Tse, "Inference of link delay in communication networks," IEEE Journal on Selected Areas in Communications, vol. 24, no. 12, pp. 2235-2248, 2006.

[10] R. Castro, M. Coates, G. Liang, R. Nowak, and B. Yu, "Network tomography: Recent developments," Statistical Science, vol. 19, no. 3 , pp. 499-517, 2004.

[11] N. Duffield, "Network tomography of binary network performance characteristics," IEEE Trans. Information Theory, vol. 52, no. 12, pp. 5373-5388, 2006.

[12] D. Donoho, "Compressed sensing," IEEE Trans. Information Theory, vol. 52, no. 4, pp. 1289-1306, 2006.

[13] E. Candès, J. Romberg, and T. Tao, "Robust uncertainty principles: Exact signal reconstruction from highly incomplete frequency information," IEEE Trans. information theory, vol. 52, no. 2, pp. 489-529, 2006.

[14] E. Candès, "Compressive sampling," in Proceedings of the International Congress of Mathematicians, vol. 3, 2006, pp. 1433-1452.

[15] M. Wakin, "An introduction to compressive sampling," IEEE Signal Pprocessing Magazine, 2008.

[16] A. Gilbert, M. Strauss, J. Tropp, and R. Vershynin, "One sketch for all: fast algorithms for compressed sensing," in Proceedings of the thirtyninth annual ACM symposium on Theory of computing, 2007, pp. 237246.

[17] E. Candes and T. Tao, "Near-optimal signal recovery from random projections: Universal encoding strategies?" IEEE Trans. Information Theory, vol. 52, no. 12, pp. 5406-5425, 2006.

[18] W. Xu and B. Hassibi, "Efficient compressive sensing with deterministic guarantees using expander graphs," in IEEE Information Theory Workshop, 2007, pp. 414-419.

[19] R. Berinde and P. Indyk, "Sparse recovery using sparse random matrices," preprint, 2008.

[20] A. Lubotzky, R. Phillips, and P. Sarnak, "Ramanujan graphs," Combinatorica, vol. 8, no. 3, pp. 261-277, 1988.

[21] A. Ta-Shma, C. Umans, and D. Zuckerman, "Lossless condensers, unbalanced expanders, and extractors," Combinatorica, vol. 27, no. 2, pp. 213-240, 2007.

[22] M. Capalbo, O. Reingold, S. Vadhan, and A. Wigderson, "Randomness conductors and constant-degree lossless expanders," in Proceedings of the thiry-fourth annual ACM symposium on Theory of computing. ACM, 2002, pp. 659-668.

[23] P. Indyk and M. Ruzic, "Near-optimal sparse recovery in the 11 norm," in IEEE 49th Annual IEEE Symposium on Foundations of Computer Science, 2008, pp. 199-207.

[24] M. H. Firooz and S. Roy, "Network tomography via compressed sensing," UW Technical Report, Feb 2010. 